\title{
Proposal for a Universal Test Mirror for Characterization of Slope Measuring Instruments
}

\author{
Valeriy V. Yashchuk, ${ }^{* a}$ Wayne R. McKinney, ${ }^{\text {a }}$ Tony Warwick, ${ }^{\mathrm{a}}$ \\ Tino Noll, ${ }^{\mathrm{b}}$ Frank Siewert, ${ }^{\mathrm{b}}$ Thomas Zeschke, ${ }^{\mathrm{b}}$ Ralf D. Geckeler ${ }^{\mathrm{c}}$ \\ ${ }^{a} A d v a n c e d$ Light Source, Lawrence Berkeley National Laboratory, Berkeley, CA 94720, USA \\ ${ }^{b}$ BESSY mbH, Albert-Einstein-Str. 15, 12489 Berlin, Germany \\ ${ }^{\mathrm{c}}$ Physikalische-Technische Bundesanstalt Braunschweig (PTB), Bundesallee 100, 38116 \\ Braunschweig, Germany
}

\begin{abstract}
The development of third generation light sources like the Advanced Light Source (ALS) or BESSY II brought to a focus the need for high performance synchrotron optics with unprecedented tolerances for slope error and micro roughness. Proposed beam lines at Free Electron Lasers (FEL) require optical elements up to a length of one meter, characterized by a residual slope error in the range of $0.1 \mu \mathrm{rad}(\mathrm{rms})$, and $\mathrm{rms}$ values of $0.1 \mathrm{~nm}$ for micro roughness. These optical elements must be inspected by highly accurate measuring instruments, providing a measurement uncertainty lower than the specified accuracy of the surface under test. It is essential that metrology devices in use at synchrotron laboratories be precisely characterized and calibrated to achieve this target. In this paper we discuss a proposal for a Universal Test Mirror (UTM) as a realization of a high performance calibration instrument. The instrument would provide an ideal calibration surface to replicate a redundant surface under test of redundant figure. The application of a sophisticated calibration instrument will allow the elimination of the majority of the systematic error from the error budget of an individual measurement of a particular optical element. We present the limitations of existing methods, initial UTM design considerations, possible calibration algorithms, and an estimation of the expected accuracy.
\end{abstract}

Keywords: slope measuring instrument, long trace profiler, LTP, calibration, systematic error reduction, test mirror

\section{INTRODUCTION}

The development of synchrotron radiation sources of the so-called 'third generation' family brought to a focus the need for high performing X-ray optics with unprecedented tolerances for surface slope error and roughness. For example, specification of the surface quality of silicon substrates for gratings designed in the frame of the MERLIN (MilliElectron-volt Resolution beamLINe) project developed by the ALS Scientific Support Group ${ }^{1}$ requires a slope error less than $0.25 \mu \mathrm{rad}(\mathrm{rms})$. Proposed beam lines for BESSY, SLAC Free Electron Lasers (FEL), and the European X-ray FEL require optical elements characterized by a residual slope error of $0.1 \mu \mathrm{rad}(\mathrm{rms})$ and even better. ${ }^{2-4}$ The same quality of $\mathrm{X}$-ray optics is desired in order to realize the performance of the National Synchrotron Light Source II (NSLS-II) that is under construction at BNL. ${ }^{5}$ However, the current performance of X-ray optical metrology instruments generally falls short of these requirements. A long trace profiler LTP-II, ${ }^{6-8}$ available at the ALS Optical Metrology Laboratory (OML), was designed in the early ninety's to have an accuracy of about $0.5-1 \mu \mathrm{rad}(\mathrm{rms})$. The Nanometer Optic component measuring Machine (NOM) $)^{9}$ developed at BESSY provides the world-best sensitivity to mirror slope measurements of $\sim 0.05 \mu \mathrm{rad}$ (rms) for a flat mirror and $\sim 0.25 \mu \mathrm{rad}$ (rms) for a significantly curved mirror. A spherical grating blank for the MERLIN-project with a radius of $\sim 15 \mathrm{~m}$ and a specific residual slope error of $0.25 \mu \mathrm{rad}$ rms was recently inspected with the NOM. The estimated measurement uncertainty for these measurement is about $0.2 \mu \mathrm{rad} \mathrm{rms} .{ }^{10}$ However, as it will be shown in Sec. 3, even after precise calibration, the NOM is still subject to systematic errors on the level of 0.2 $\mu \mathrm{rad}$ (for a view field of $\pm 500 \mathrm{arcsec}$ ) to $0.5 \mu \mathrm{rad}$ (for a view field of about $\pm 1000 \mathrm{arcsec}$ ).

*vvyashchuk@lbl.gov; tel +1-510-495-2592; fax +1-510-486-7696 
In Ref. ${ }^{11}$ a new method for calibration of slope measuring instruments has been proposed. In the present work we describe the basis of the calibration method, show its capability to potentially overcome the problems of the systematic errors of the LTP and NOM, and concentrate on design specifications and basic design requirements for a Universal Test Mirror (UTM) which realizes the method.

\section{SYSTEMATIC ERRORS AND REQUIREMENTS FOR PRECISE CALIBRATION}

Since the development of the first LTP based on a pencil-beam interferometer, ${ }^{6-8}$ investigations to determine the LTP limiting factors and to find the ways to overcome them have been carried out at practically all facilities ${ }^{12-27}$ It should be mentioned that most sources of systematic errors and random noise are common for the LTP and for the principally different slope profilers $\mathrm{NOM}^{9}$ and ESAD (Extended Shear Angle Difference Shearing Deflectometer ${ }^{28}$ ) based on a high precision autocollimator and developed at BESSY and the PTB, respectively. Compared to the classical LTP-II the progress of $2^{\text {nd }}$ generation slope measuring instruments like the NOM and the ESAD is based on an advanced design of the instruments and high stability of their environmental conditions. The main goal was to suppress the random noise and, if it is possible, the systematical errors. High stability of the instruments allows for averaging the random noise, providing excellent reproducibility of the measurements. Thus, a precision calibration can be applied to identify the systematic errors.

In recent work ${ }^{26,27}$ performed at the ALS Optical Metrology Laboratory (OML), it was demonstrated that the major random noise arises due to perturbation of direction (pointing stability) of the LTP laser beam due to pointing instability of the diode laser itself, and due to thermal air convection along the beam optical path. The first problem can be solved by using a light source based on a fiber coupled diode laser. Such a laser supplied by Melles Griot Inc. ${ }^{29}$ was tested at the

OML and found to have a pointing stability on the level of $0.1 \mu \mathrm{rad} / \sqrt{\mathrm{Hz}}(\mathrm{rms})$. A fiber-coupled He-Ne laser is used for the LTP arm of the NOM and has shown a high pointing stability as well.

Air convection noise is especially pronounced in the LTP-II reference channel due to the long reference-beam optical path, which also varies in the course of measurement. In order to suppress the noise, an air-blowing method has been developed and tested at the ALS OML ${ }^{27}$ The method allows one to control the spectral characteristics of the noise, and to suppress the low frequency part of the noise, which is most important from the point of view of LTP measurements.

An additional improvement of the level of the LTP random noise is possible if a precise tiltmeter is used instead of (or additionally to) the optical reference channel. Experimental tests performed at the OML with a tiltmeter (Geomechanics Inc. ${ }^{30}$ ) placed on the carriage of a specially designed linear translation stage has demonstrated a capability of the tiltmeter to monitor the wobbling and wiggling of the carriage with accuracy of $\sim 0.1 \mu \mathrm{rad}(\mathrm{rms}) .{ }^{31}$ However, the tiltmeter was found to be extremely sensitive to horizontal acceleration ${ }^{32}$ and cannot be used with original LTP-II, measuring mode with a continuously moving carriage. The tiltmeter-based reference can be realized with the modernized LTP-II available now at the OML. ${ }^{33}$ Here, the slope measurements are carried out after the carriage makes a discrete move, so called start/stop mode.

It should be specially mentioned that the stability of an optical profiler system, as well as the intensity of the air convection and performance of the tiltmeter are strongly correlated with environmental conditions in the lab. ${ }^{31}$ Currently, the ALS OML air-conditioner keeps the room temperature stable on the level of $\sim 20^{\circ} \mathrm{C}$ with about 100 -mK periodic variation with approximately 10-min period determined by the switch-on/switch-off circle of the conditioner. This temperature variation is seen as a $20-\mathrm{mK}$ variation inside an instrument hutch at the OML. That causes, for example, a synchronous temperature variation of the tiltmeter reading of approximately 0.1 to $0.2 \mu \mathrm{rad}$. Careful control of the experimental environmental conditions (high temperature stability, low humidity, absence of air turbulence) is also crucial for successful operation of profilers with movable pentaprisms, such as the NOM and ESAD. For this reason, the NOM system is surrounded with a two-layer thermally-isolating hutch, providing stability of the temperature of 2 to 3 $\mathrm{mK}$ per hour.

In any case, with the listed developments (a fiber-coupled diode laser with high pointing stability; air-blowing for suppression of air convection noise; a tiltmeter-based reference channel together with a more stable linear translation system; an appropriate improvement of the laboratory environmental conditions) it seems to be possible to decrease the LTP random noise down to the level of below $0.1 \mu \mathrm{rad}$ (rms). Such (and even lower) random noise $(\sim 0.01 \mu \mathrm{rad})$ of the NOM measurements has been achieved with multiple measurements of a surface under test with subsequent averaging of the obtained traces. 
Compared to the random noise, the systematic errors of a slope profiler present significantly more difficult problems and require development of more sophisticated approaches to overcome them. The systematic errors can be divided into two groups. One group relates to the quality of the optical elements (e.g., optical homogeneity of materials used for the beam splitters, Fourier transform lens, Dove prism, and quality of the reflecting surfaces of the mirrors and mirror prisms) at higher spatial frequency. The systematic errors related to the LTP detector system ${ }^{26}$ can also be attributed to this group. In a slope trace measured with the LTP-II or the NOM, these systematic errors appear as local (high special frequency) perturbations with amplitude up to $1 \mu \mathrm{rad}$ (see also Figs. 2 and 3). Besides the improvement of quality of the optical elements, there is another way to decrease the contribution due to the high spatial frequency systematic errors. Namely, one should perform multiple measurements with the same optic under metrology, but with a different alignments and orientations with respect to the LTP laser optics. For such measurements, the systematic perturbation would appear at different places of the slope trace and could be reduced by averaging over the measurements. Practically, with averaging, the high frequency systematic errors of the LTP-II can be suppressed to the level of approximately 0.3 to $0.4 \mu \mathrm{rad} .{ }^{33} \mathrm{~A}$ non-trivial experimental issue is how to recognize the residual systematic errors after averaging. Some improvement is possible with straightforward replacement of the optical elements with ones with better performance; but it can be rather expensive. As to the systematic errors associated with the LTP detector, they can be decreased to a level of below $0.1 \mu \mathrm{rad}$ with an appropriate choice of a CCD camera and its careful calibration. ${ }^{26,33}$

Another group of systematic errors, namely low-spatial-frequency ones, relates to the uncertainty of the LTP calibration, such as unaccounted nonlinearity of the LTP position-to-slope conversion. Indeed, the LTP, as well as the NOM, dynamic range runs almost over five orders of magnitude, from a few tenths of a microradian to ten milliradians. However, a simple calibration with a diffraction grating (this is the method used routinely at the $\mathrm{OML}^{34}$ ) provides calibration only for a particular and very limited part of the dynamic range. A more sophisticated calibration method has been developed at BESSY ${ }^{35,36}$ (see also the next section). The method is based on use of a test flat mirror mounted on a precise tilting stage. The tilt of the mirror is controlled with two high precision displacement sensors and at the same time is measured with the profiler, allowing for partial accounting of the non-linearity. The account is partial because it is performed at one certain position of the test mirror in respect to the profiler optical elements. Note also that for the same reason, the calibration does not solve the problem of high frequency systematic errors.

\section{LIMITATIONS OF THE EXISTING CALIBRATION METHODS}

Currently, the best performance of calibration of a slope measurement instrument was obtained with a mirror placed on a precise tilting stage. ${ }^{35-37}$ Such a calibration set-up developed at BESSY is shown in Fig. 1, as it is installed on the NOM optical table. The tilting stage has an aluminum bar with U-shaped section of approximately $1300-\mathrm{mm}$ length and $\sim 120 \mathrm{~mm}$ width for placing a test mirror at the desired position under the movable pentaprism of the NOM, with a collimation diaphragm. The bar can be precisely tilted around the axis under the center of the bar. The axis is formed with a structure of two perpendicular flexures made of a thin sheet of beryllium bronze. Control for the tilting is performed with a stepper motor ${ }^{38}$ and two displacement sensors ${ }^{39}$ mounted on the opposite ends of the bar. The sensors have resolution of about $5 \mathrm{~nm}$ providing the tilting resolution of approximately $10 \mathrm{nrad}$. The base distance $(\sim 1247 \mathrm{~mm})$ of the tilting stage was precisely measured with a ZEISS-CMM. The accuracy of the tilting stage is estimated to be approximately $50 \mathrm{nrad}(\mathrm{rms}){ }^{37}$

With the calibration system shown in Fig. 1, calibration of the LTP and NOM can be performed over the entire slope measurement range, and at various positions along the length of the instrument by moving the test mirror repeatedly to one specific position underneath the profiler optical elements as they are moved along on the carriage. Such a calibration allows the correction of a significant part of the systematic errors of the instrument. ${ }^{36,37}$

However, the calibration is not absolute. Figure 2 illustrates the statement with the calibration curves obtained at different positions of the calibration mirror. In Fig. 2, a calibration curve depicts the difference between the slope angle measured with the NOM and the tilting angle of the calibration mirror as a function of the mirror tilting angle. Each calibration curve is a result of averaging over a minimum of 6 sequential cycles of measurement at increasing and decreasing the mirror tilt angles. In the course of taking data, the position of the mirror was changed manually with following realignment of the mirror. The total range of mirror displacement was $890 \mathrm{~mm}$.

In the range of tilting angles between $-500 \operatorname{arcsec}(-2.4 \mathrm{mrad})$ and $+500 \operatorname{arcsec}(+2.4 \mathrm{mrad})$ in Fig. 2 , the calibration curves obtained at the different mirror positions overlap with an accuracy of the higher frequency systematic variations of each individual calibration. The variations are on the level of approximately 0.2 to $0.5 \mu \mathrm{rad}$, while the random 
fluctuations are estimated to be on the level of $0.05 \mu \mathrm{rad}(\mathrm{rms})$. Note that in this intermediate range of tilting angles, the systematic variations of the green and black curves measured at the same position of the calibration mirror $(590 \mathrm{~mm}$ that is approximately at the center of the NOM optical table), are almost coincident. This can be thought of as an evidence for the systematic character of the variations. The observed dependence of the variations on the mirror position can be attributed to the properties of the NOM rather than to the properties of the calibration system. The green and black curves in Fig. 2 were obtained at different times and at different alignments of the calibration mirror. This can be a reason for a difference of approximately $0.5 \mu \mathrm{rad}$ between the curves at the higher tilting amplitudes.

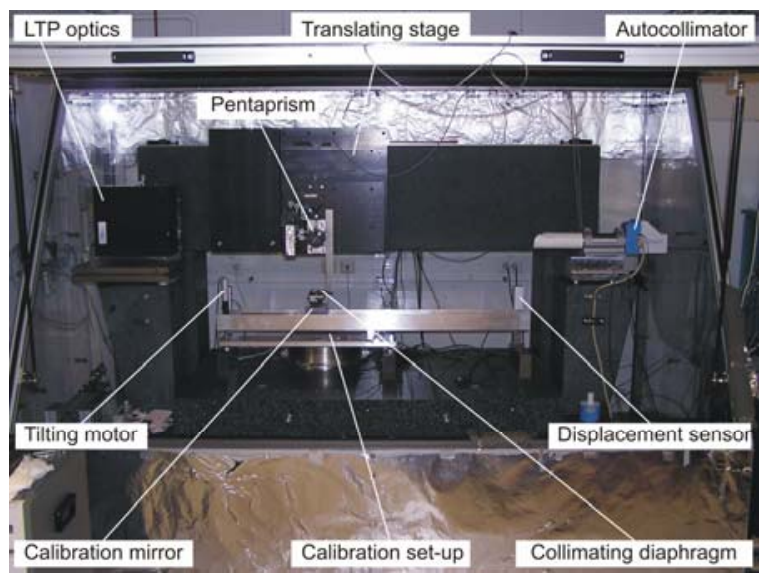

Figure 1: BESSY calibration set-up installed for calibration of the NOM profiler.

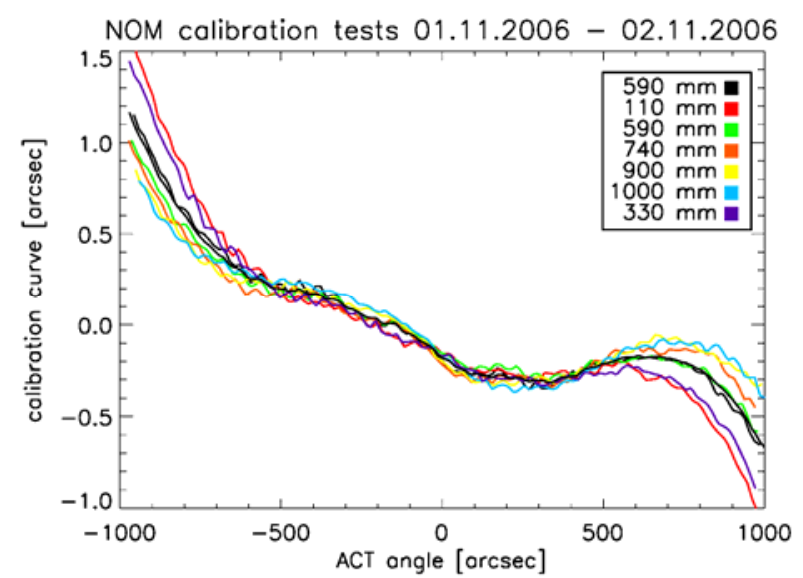

Figure 2: the calibration curves obtained at different positions of the calibration mirror. A calibration curve represents the difference between the slope angle measured with the NOM and the tilting angle of the calibration mirror as a function of the mirror tilting angle.

Note that the difference between the calibrations measured at tilting amplitudes larger than $\sim 2.4$ mrad is significantly larger, up to $2.5 \mu \mathrm{rad}$ that can be due to a systematic effect specific to the NOM, namely, the effect of misalignment of the NOM light beam with respect to the collimating aperture appeared at larger tilting angles. This question is under more detailed investigation.

The usual procedure of a NOM calibration uses a calibration function, which is a firth/sixth order polynomial function best fitted to the calibration curve measured with the calibration mirror placed at the position of the center of the surface under test. In Ref. ${ }^{36}$ the calibration curve obtained with the calibration mirror was used, as it is, to correct the measured slope profile. In order to improve the correction for measurements with a significantly curved surface, a calibration curve obtained by averaging of 3 curves, taken at the edge and center positions of the line inspected, was used. ${ }^{37}$ Such a calibration was shown to provide an accuracy of $<0.5 \mu \mathrm{rad}(\mathrm{rms})$ while measuring curved x-ray optics at BESSY, e.g. rotationally symmetric ellipsoids, with lengths up to $300 \mathrm{~mm}$. Figure 3 shows traces of the residual systematic errors after the calibration. The plots in Fig. 3 were obtained with subtraction from each calibration in Fig. 2 of a fifth order polynomial best fitted to the result of averaging over all seven calibrations. It is seen from the spread of the plots in Fig. 3 that even in the central region of the tilting range, the error can be 0.2 to $0.3 \mu \mathrm{rad}$. At higher slope angles, the systematic error of the calibration appears to be much larger, up to $2 \mu \mathrm{rad}$.

It was also found that the NOM calibration depends significantly on position (in the vertical direction) of the collimating diaphragm and calibration mirror. The discrepancy of the calibration curves, when the mirror and the diaphragm were raised up by $\sim 50 \mathrm{~mm}$, was as large as 2 to $3 \mu \mathrm{rad}$ at large tilting amplitudes and 0.2 to $0.5 \mu \mathrm{rad}$ at the intermediate range of the tilting angle. The observed discrepancy of the calibration curves measured at the different positions of the calibration mirror and the collimating diaphragm is clear evidence for the strongly required necessity of a more rigorous calibration method in order to allow high precision slope measurements with accuracy better than 0.2 to $0.3 \mu \mathrm{rad}$. An idea for such a calibration method suggested in Ref. ${ }^{11}$ is presented in the next section. 


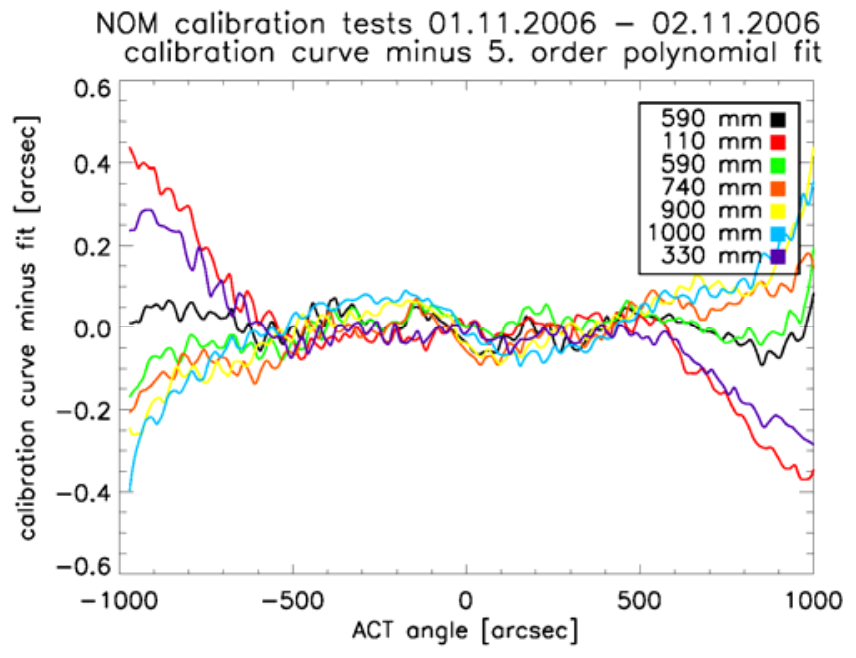

Figure 3: The distributions of the residual systematic errors for after calibration of the NOM with a fifth order polynomial function best fitted to the calibration averaged over all seven calibrations shown in Fig. 2.

\section{CONCEPT OF A UNIVERSAL TEST MIRROR AND OPTIMAL CALIBRATION ALGORITHM}

As it was shown in the previous section, in order to succeed, a calibration method has to provide a calibration of a profiler at all possible arrangements. In the case of the NOM, these are all the different positions of the movable optics in the horizontal and in the vertical directions, leading to a multi-dimensional calibration matrix with, basically, huge number of elements $a_{i, j, k}$, where the index $i$ runs over all possible tangential positions; the index $j$ accounts possible position of the diaphragm; and the index $k$ covers the interesting range of measuring angles.

A significantly more efficient calibration method would be possible, if for each mirror under test, there would be a specific test mirror with an 'ideal' quality and with a shape, absolutely identical to the shape of the surface under test (SUT). An additional measurement with such an ideal test mirror at an experimental arrangement, corresponding to the measurement with the SUT, would provide a precise calibration valid for this particular surface under test. In spite of the apparent idealization that this concept merely reduces the measurement to a comparison or transfers from a non-existent ideal mirror, a method and its realization described below can potentially provide such an opportunity.

Figure 4 schematically illustrates the idea of the proposed calibration method. The dashed-line box depicts a standard LTP-II arrangement ${ }^{13}$ with an optical head mounted on a linearly translated carriage. The set-up shown in Fig. 4 as a mirror to be measured is a universal test mirror (UTM) proposed here. The UTM set-up includes a linear stage with a carriage translatable over a distance corresponding to the maximum mirror length measurable with the LTP. The UTM main element is a flat test mirror with the best available quality. The mirror is mounted on a precise tilting stage. There is also a closed-loop control system for the mirror tilt based on monitoring of the tilting angle with a high precision autocollimator and a tiltmeter. The system must be able to provide a controllable tilt with accuracy of $<0.1 \mu \mathrm{rad}$. Therefore, the automated control for translation and tilt of the mirror would allow simulation of a reflecting surface with any possible figure. For our mathematical notation we will use abbreviation DF for "defined figure."

Now let us consider an algorithm of measurement of a mirror under test using the UTM system described above.

1. First, a regular LTP measurement of a slope trace $\alpha_{\text {MIRR }}(x)$ with the mirror has to be performed with all necessary precautions for decreasing the random noise to the level below the desired accuracy of the metrology. The trace $\alpha_{\text {MIRR }}(x)$ obtained at this point can be thought of as a sum of a slope trace of the surface under test (SUT) that would be measured with an ideal LTP, free of any systematic errors, $\alpha_{S U T}(x)$, and a trace of systematic distortions, $\alpha_{S Y S}^{M}(x)$, where superscript $M$ denotes that the trace corresponds to a measurement with the mirror under test:

$$
\alpha_{\text {MIRR }}(x)=\alpha_{\text {SUT }}(x)+\alpha_{S Y S}^{M}(x) .
$$




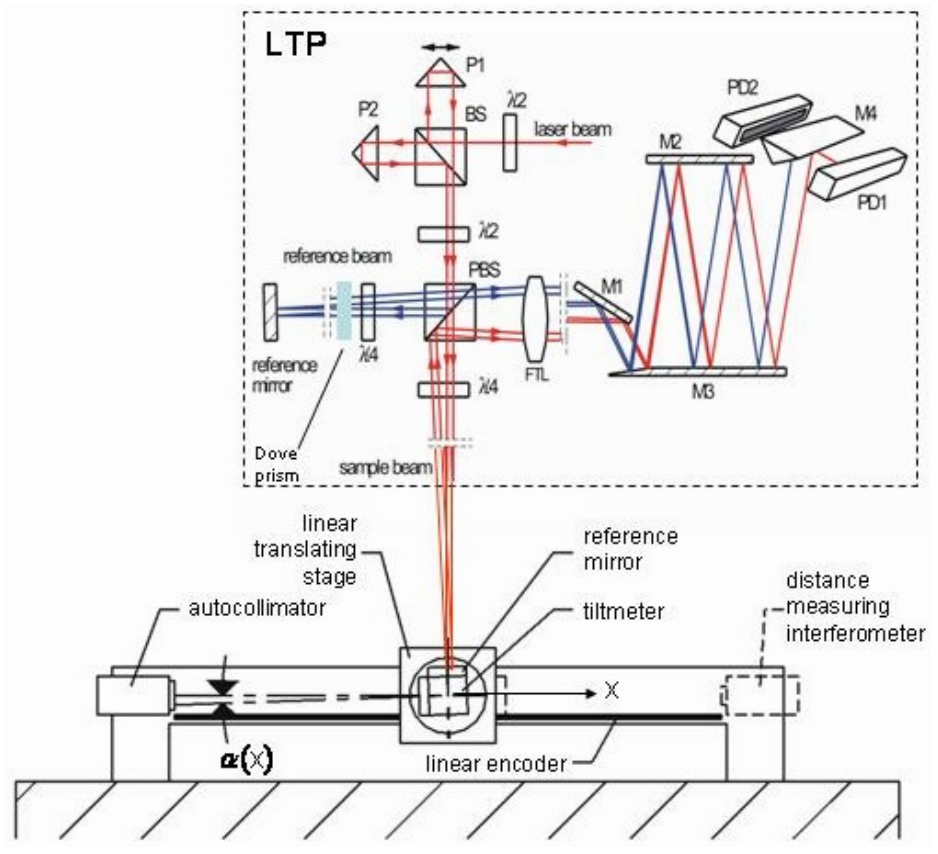

Figure 4: Schematic illustration of the idea of the proposed calibration method. The dashed-line box depicts a standard LTP-II arrangement ${ }^{13}$ with an optical head mounted on a linearly translated carriage. The red lines correspond to the test (sample) beam; the blue lines show the reference beam. The LTP reference mirror is posted on the same base (optical table) as a mirror under measurement. The set-up of the universal test mirror is shown to be placed as a mirror under test.

Note that this is the only measurement with the mirror under test which we need. All other measurements described below are performed with the UTM. We assume that these measurements are also performed with the precautions necessary in order to decrease the random noise to a level below the desired accuracy of the metrology.

The basic idea of the measurements with the UTM is to find by iteration a "defined figure" for scanning the UTM such that the corresponding trace measured with the LTP exactly coincides with the trace $\alpha_{\text {MIRR }}(x)$ measured with the mirror under test. Obviously, this final, after $N$ iterations, UTM figure $\alpha_{D F, N}(x)$ will represent the SUT slope trace $\alpha_{S U T}(x)$ that would be measured with an ideal LTP, free of any systematic errors:

$$
\alpha_{D F, N}(x) \approx \alpha_{S U T}(x) .
$$

2. On the first iteration step, a slope trace $\alpha_{\text {MIRR }}(x)$ is used as a DF for UTM scanning:

$$
\alpha_{D F, 1}(x)=\alpha_{M I R R}(x)
$$

3. Simultaneously and synchronously scanning the UTM and the LTP, the first test trace, $\alpha_{T E S T, 1}(x)$, is measured. The trace $\alpha_{T E S T, 1}(x)$ can be thought of as a sum of the DF function $\alpha_{D F, 1}(x)$ used at this point and a trace of systematic distortions, $\alpha_{S Y S, 1}^{T}(x)$, where superscript $T$ denotes that the distortion trace corresponds to measurement with the universal test mirror:

$$
\alpha_{T E S T, 1}(x)=\alpha_{D F, 1}(x)+\alpha_{S Y S, 1}^{T}(x) .
$$

On the other hand, the first test measurement can be presented as a measurement of the DF figure with the systematic error [two terms in the square brackets in Eq. (5)], corresponding to the SUT surface, plus systematic error $\alpha_{S Y S}^{M}(x)$ of the measurement of $\alpha_{S U T}(x)$, and an additional perturbation term $\delta \alpha_{S Y S, 1}^{M}(x)$, appearing due to the deviation of the used DF from the SUT figure:

$$
\alpha_{T E S T, 1}(x)=\left[\alpha_{S U T}(x)+\alpha_{S Y S}^{M}(x)\right]+\alpha_{S Y S}^{M}(x)+\delta \alpha_{S Y S, 1}^{M}(x) .
$$


Because practically ${ }^{*}$ the magnitude of the systematic error is much smaller than the magnitude of the surface slopes, $\alpha_{S Y S}^{M}(x)<<\alpha_{S U T}(x)$, the additional systematic error term $\delta \alpha_{S Y S, 1}^{M}(x)$ has to be relatively small (we are assuming the error is additive for small changes),

$$
\delta \alpha_{S Y S, 1}^{M}(x)<<\alpha_{S Y S}^{M}(x) .
$$

Therefore,

$$
\alpha_{T E S T, 1}(x) \approx \alpha_{S U T}(x)+2 \alpha_{S Y S}^{M}(x) .
$$

Comparing (3), (4), and (7), one can see that the systematic error measured with UTM is a good approximation to the systematic perturbation for the measurement of the SUT figure:

$$
\alpha_{S Y S, 1}^{T}(x)=\alpha_{T E S T, 1}(x)-\alpha_{D F, 1}(x) \approx \alpha_{S Y S}^{M}(x) .
$$

4. For the second iteration, the DF function is chosen to be closer to the SUT figure:

$$
\begin{aligned}
\alpha_{D F, 2}(x) & =\alpha_{M I R R}(x)-\alpha_{S Y S, 1}^{T}(x) \\
& =\alpha_{S U T}(x)+\delta \alpha_{S U T, 2}(x),
\end{aligned}
$$

where $\delta \alpha_{S U T, 2}(x)$ gives the difference between the second DF function and the SUT figure.

5. Similar to (4) and (5), the second measurement with the UTM gives:

$$
\begin{gathered}
\alpha_{T E S T, 2}(x)=\alpha_{D F, 2}(x)+\alpha_{S Y S, 2}^{T}(x)=\alpha_{M I R R}(x)-\alpha_{S Y S, 1}^{T}(x)+\alpha_{S Y S, 2}^{T}(x), \\
\alpha_{T E S T, 2}(x)=\alpha_{S U T}(x)+\delta \alpha_{S U T, 2}(x)+\alpha_{S Y S}^{M}(x)+\delta \alpha_{S Y S, 2}^{M}(x) .
\end{gathered}
$$

6. Combining (10) and (11), one can find the error of the approximation (9):

$$
\delta \alpha_{S U T, 2}(x)=\alpha_{S Y S, 2}^{T}(x)-\alpha_{S Y S, 1}^{T}(x)+\delta \alpha_{S Y S, 2}^{M}(x) \approx \alpha_{S Y S, 2}^{T}(x)-\alpha_{S Y S, 1}^{T}(x) .
$$

The rms variation of the error trace $\delta \alpha_{S U T, 2}(x)$ provides a measure for the convergence of the iteration procedure.

7. Expression (12) can be rewritten in the form:

$$
\alpha_{S Y S, 2}^{T}(x)=\alpha_{S Y S, 1}^{T}(x)+\delta \alpha_{S U T, 2}(x)-\delta \alpha_{S Y S, 2}^{M}(x) ;
$$

and after that Eq. (13) can be used to find the DF function for the next iteration [compare with (9)]:

$$
\begin{aligned}
\alpha_{D F, 3}(x) & =\alpha_{M I R R}(x)-\alpha_{S Y S, 2}^{T}(x) \\
& =\alpha_{M I R R}(x)-\alpha_{S Y S, 1}^{T}(x)-\delta \alpha_{S U T, 2}(x)+\delta \alpha_{S Y S, 2}^{M}(x) \\
& =\alpha_{S U T}(x)+\delta \alpha_{S Y S, 2}^{M}(x) .
\end{aligned}
$$

Because for $\delta \alpha_{S Y S, 2}^{M}(x)$ we should expect to be true a relation analogous to (6):

\footnotetext{
* This assumption does not work for a flat SUT. However, in this case during slope trace measurement, the LTP sample beam is passing along the same optical path inside the optics, instead of walking across the optics as it is in the case of a curved SUT. For this steady path, the LTP can be more easily calibrated, e.g. with a grating based method. Moreover, the systematic effects due to imperfections of the optics would be also negligible. The real problem for the case of a flat SUT still is the stability of environmental conditions, associated drifts of the LTP. A further source of error affecting the measurement uncertainty in this case is the so called sub-pixel error of the photo-detector. Especially in the case of old LTP-II instruments compared to state of the art instruments, CCDs show significant inhomogeneity regarding pixel spacing and sensitivity of the single pixels. Detection of this type of error source have been shown by measurements at the PTB and at BESSY by use of different calibration instruments; see also. ${ }^{26}$
} 


$$
\delta \alpha_{S Y S, 2}^{M}(x)<<\alpha_{S Y S, 2}^{T}(x) \approx \alpha_{S Y S, 1}^{T}(x),
$$

the expression (15) can be interpreted as a proof of the convergence of the iteration procedure.

In principle, the test measurements with the UTM can be finished at this point and the slope trace $\alpha_{D F, 3}(x)$ determined by (14) can be used as the sought-for SUT figure free of the LTP systematic error on the level of the second order correction given by (15). However, an additional measurement can be performed in order to verify the convergence of the procedure and to estimate the accuracy of the found correction.

If the accuracy turns out to be not good enough, the next iteration with the DF function $\alpha_{D F, 3}(x)$ has to be performed. As a result of this iteration the next DF function

$$
\alpha_{D F, 4}(x)=\alpha_{M I R R}(x)-\alpha_{S Y S, 3}^{T}(x)
$$

would be found. A deviation $\delta \alpha_{S Y S, 3}^{M}(x)$ of the $\alpha_{D F, 4}(x)$ from the SUT figure $\alpha_{S U T}(x)$ is expected to be a small correction of $\delta \alpha_{S Y S, 2}^{M}(x)$.

\section{DESIGN SPECIFICATION FOR A UNIVERSAL TEST MIRROR}

In order to realize the potential advantages of the proposed method, a UTM has to satisfy to rather tight specification. Here we consider just the basic requirements and tolerances of a UTM tilting stage that can be used as a starting point for engineering consideration and design of a UTM.

A UTM tilting stage should provide the possibility for closed-loop control of the tilting of a calibration mirror with an accuracy of $<0.05 \mu \mathrm{rad}$ at the total range of tilting angles of $\pm 20 \mathrm{mrad}$. A larger range, up to $\pm 50 \mathrm{mrad}$, is desired for a capability to calibrate a slope profiler instrument for stitching measurements of greatly curved surfaces.

The stage has to provide a tilt around an axis adjustable in two directions, as it is shown in Fig. 5. This requirement comes from the experience of using the BESSY calibration system. The accuracy of the adjustment has to be better than $20 \mu \mathrm{rad}$ for both adjustments.
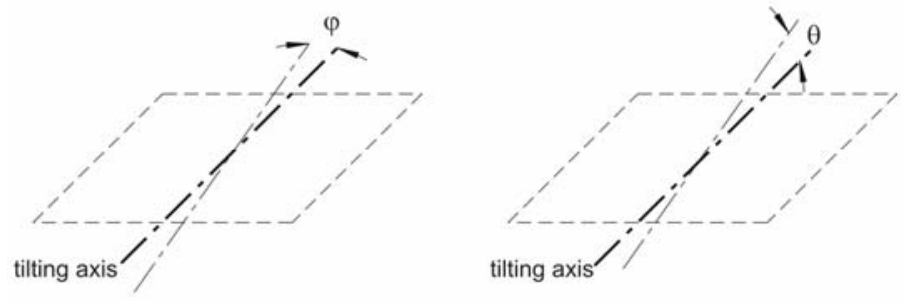

Figure 5: Illustration of a requirement for precise adjustment of the axis of the tilting stage.

Control for tilting of the calibration mirror can be performed with two drivers: one driver (e.g., a stepper motor) for rough tuning of the angle to be as close to the desired angle as 2 to $5 \mu \mathrm{rad}$; and another driver (e.g., a piezoceramic actuator) to precisely tune the tilting angle from $\sim 5$ to $0.05 \mu \mathrm{rad}$ to compensate a mistuning of the rough driver.

The possible schematics of a UTM system with one reference mirror and one autocollimator used to control the tilting angle are depicted in Fig. 6. The first arrangement corresponds to the two-reference-mirror schematic with the right arm cut out. The advantages of the schematic are the same as ones mentioned for the case of two reference mirrors, excepting the symmetry of the angle control. Symmetry of the design can be maintained with a second dummy reference mirror. Such a system can be easily upgraded to the two mirror arrangement if it is found to be desirable. The second schematic based on a reference mirror placed beyond the calibration mirror is free of the disadvantage of previous arrangements related to the sticking up edge of the reference mirror. However, the price for that is an additional source of a systematic error due to a dependence of the distance between the autocollimator and reference mirror surface on the tilting angle. A geometrical layout of the systematic error is presented in Fig. 7. 

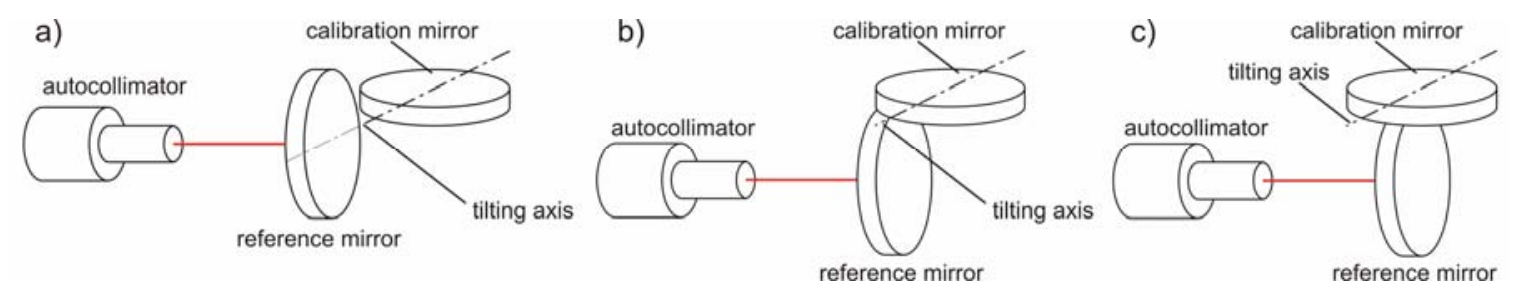

Figure 6: UTM schematics with one reference mirror and one autocollimator.

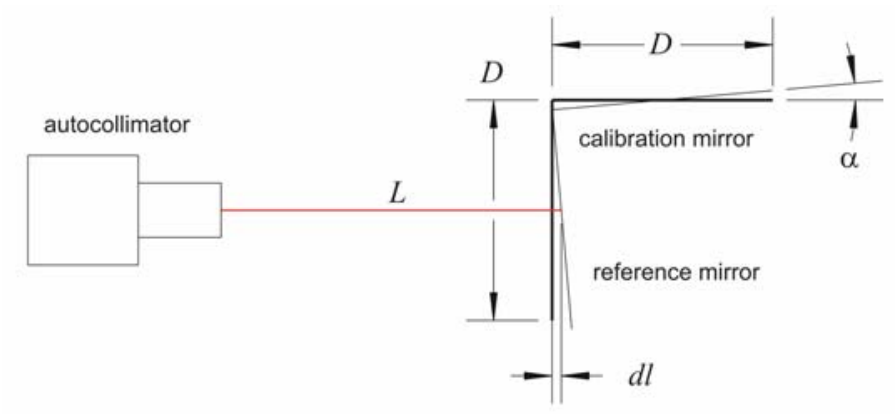

Figure 7: Geometrical layout of a UTM schematic with one reference mirror and one autocollimator shown in Fig. 6 b.

It is easy to see, that the autocollimator-reference mirror distance, $L$, is a function of the tilting angle, $\alpha$,

$$
L(x, \alpha)=L(x, 0)+d l(\alpha) \approx L(x, 0)+\alpha D / 2 .
$$

At $\alpha=50 \mathrm{mrad}$ (the desired maximum amplitude of tilting for the stage), displacement is about $d l \approx \alpha D / 2 \approx 1.9 \mathrm{~mm}$ at $D=75 \mathrm{~mm}$ and that can produce a significant systematic effect. Note that in this case, calibration of a UTM itself would be a challenging problem.

A UTM based on the arrangement shown in Fig. $6 \mathrm{~b}$ will have one more problem due to the vertical displacement of the reference mirror at nonzero tilting angles. The displacement has the same dependence on the tilting angle as depicted with Eq. (17) with maximum value about $2 \mathrm{~mm}$. This effect can potentially produce a systematic error due to a deviation of the reference mirror surface from a flat shape. The problem can be solved by placing the reference mirror with the working surface directly below the axis of rotation as it is shown in Fig. 6c.

We are working now on an effective yet reasonably priced design for a UTM system which includes the design factors presented here, and also further considerations.

\section{DISCUSSION AND CONCLUSIONS}

The potential of the proposed UTM device for calibration and test of high precision metrology instrumentation is significantly broader than just the measurement of the systematic errors with the algorithm discussed in Sec. 4 .

In principle, such a device can be used for the measurement of a multi-parametric calibration of an instrument. In the simplest realization, which could partially solve the calibration problems discussed in Sec. 3, the multi-parametric calibration would be a two-dimensional matrix; one dimension is the position along the NOM; another dimension is the tilting angle of the calibration mirror. In this case, the matrix elements are equal to the calibration value measured at a certain position and tilt angle of the calibration mirror. The next level of complication would be including in consideration even more parameters, e.g. position of the collimating diaphragm. Necessarily, such a calibration will require cubically longer time compared with a one-dimensional calibration procedure considered in Sec. 4 . The advantage is the universality of the calibration. Efficiency of the multi-parametric calibration can be significantly increased (at the cost of universality), if the calibration is performed over a range of the tilting angles reduced to a small range of interest in the vicinity of a SUT slope angle measured at a particular position. 
Another interesting possibility is the use of the UTM device for characterizing a slope profiler in the spatial-frequency domain, rather than in the spatial domain. In this case, the DF function has to be chosen to simulate a periodic harmonic surface figure. In particular, the higher spatial frequency roll-off of a ratio of the measured amplitude of periodical variations of the slope to the amplitude of the applied DF function would provide a measure of the Modulation Transfer Function (MTF) of an instrument and its spatial frequency bandwidth for slope measurements.

A UTM system with a range of tilting angles, larger than the working range of that the LTP or the NOM, would be extremely useful when calibrating a profiler used to measure a mirror with curvature exceeding the instrument measurement range. In this case of stitching measurements, a curved calibration mirror has to be used with a UTM device in order to avoid an additional systematic error due to the difference of the shape of the SUT mirror and the UTM calibration mirror.

The present article describes the idea of a universal test mirror and proves its potential high efficiency for testing and calibration of long trace profilers with some possible calibration algorithms. In this respect, the design schematic shown in Fig. 4 is only for illustration. We are additionally analyzing more sophisticated schematics, e.g., as the one shown in Fig. 8 .

The design concept depicted in Fig. 8 is optimized to minimize errors of the reference autocollimator as in the approach shown in Fig. 4. Indeed, a common problem of slope measuring profilers is change of the optical path in the course of measurement. In order to minimize such an error while measuring with an autocollimator, the instruments should be calibrated and utilized under the same measurement conditions (working distance, lateral position, etc.).

Unlike Fig. 4, the schematic in Fig. 8 uses a linear translation stage placed on top of a precise tilting stage. Two autocollimators, one placed on the tilting stage, and another one mounted on the optical table, allow control over errors of the translation stage and the tilting stage, respectively and independently. Because wobbling and wiggling of the linear stage are supposed to be rather small $(\leq 10 \mu \mathrm{rad})$, the calibration error of the first autocollimator, possible due to change of the optical path, would negligibly affect the measurements. The second autocollimator measures the entire range of tilting angles. Its calibration error is minimized because the instrument is mounted to be the same distance from the corresponding reference mirror.

These or other schematics to be used for the UTM have to be carefully analyzed. Based on the results of the analysis, the final design of the optimized UTM system will be developed and UTM systems will be fabricated for the ALS, BESSY, and PTB.

Our understanding that the UTM system required a state-of-the-art mechanical design and very precise manufacturing, brought us to work together on the project, uniting our experiences, resources, and capabilities. However, the collaboration is open for creative collaboration with everyone interested in the development and use of the UTM technique.

The collaboration assumes that the developed UTM design will be available for the entire optical metrology community under the assumption that all further developments of the UTM system must be available for all users of the UTM technique.

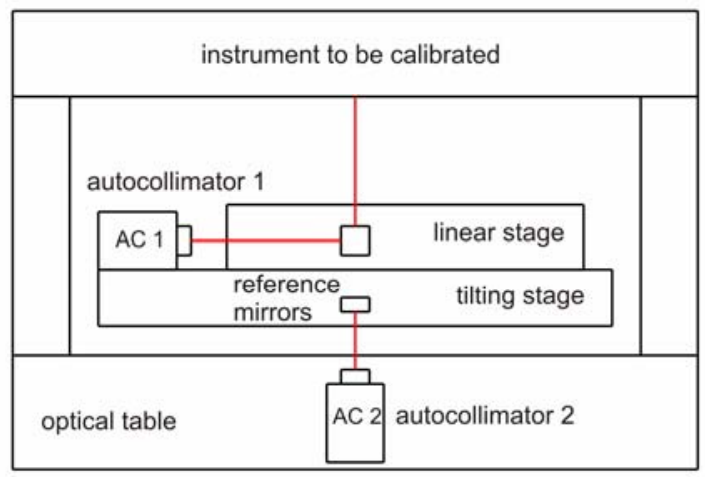

Figure 8: Possible UTM schematic with linear translation stage placed on the tilting (rotational) stage. Two autocollimators allow independent monitoring of the stages for the errors. 


\section{ACKNOWLEDGEMENTS}

The authors are grateful to D.E. Echizenya, Rob Duarte, Steve Irick, Nicholas Kelez, Jonathan Kirschman, Alastair MacDowell, Howard Padmore, and Peter Takacs for useful discussions. V.V.Y. wishes to particularly acknowledge BESSY, and its staff, for their collaboration, kind hospitality, and encouragement during his visit of October-November 2006, when much of this work was accomplished, and measurements taken with the NOM. This work was supported by the U. S. Department of Energy under contract number DE-AC02-05CH11231.

\section{DISCLAIMER}

Certain commercial equipment, instruments, software, or materials are identified in this document. Such identification does not imply recommendation or endorsement by the US Department of Energy, LBNL or ALS nor does it imply that the products identified are necessarily the best available for the purpose.

\section{REFERENCES}

1. Beamline 4.0.1: High-Resolution Spectroscopy of Complex Materials (MERLIN); www-als.lbl.gov/als/techspecs/ bl4.0.1.html.

2. D. Krämer, E. Jeschke, W. Eberhardt, "The BESSY Soft X-ray Free Electron Laser - Technical Design Report, March 2004, Berlin; www.bessy.de/publicRelations/publications.

3. R. Follath, "Design of FEL-Beamlines for Short Pulse, High Resolution and High Power Density," AIP Conference Proceedings 879 (2007).

4. A. Aghababyan , et. al., "The European X-Ray Free-Electron Laser - Technical Design Report," Editors: M. Altarelli, et. al.; http://xfel.desy.de/tdr/index_eng.html.

5. National Synchrotron Light Source II, www.bnl.gov/nsls2.

6. E. L. Church, P. Z. Takacs, "Use of an optical profiling instrument for the measurement of the figure and finish of optical quality surfaces," Wear, 109 (1986), 241-57.

7. P. Z. Takacs, Shinan Qian, J. Colbert, "Design of a long trace surface profiler," SPIE Proc. 749 (1987), 59-64.

8. P. Z. Takacs, S. K. Feng, E. L. Church, S. Qian, and W. Liu, "Long trace profile measurement on cylindrical aspheres," SPIE Proc. 966, 354 (1989).

9. F. Siewert, T. Noll, T. Schlegel, T. Zeschke, and H. Lammert, "The Nanometre Optical Component Measuring Machine: a new Sub-nm Topography Measuring Device for X-ray Optics at BESSY," AIP Conference Proceedings, 705, p.847-850 (2004).

10. F. Siewert, "BESSY Measurement Report for a Water Cooled Spherical Grating Blank of the MERLIN-Beamline at the Advanced Light Source," BESSY, Berlin (2007).

11. V. V. Yashchuk, "Universal Test Mirror for Calibration of Long Trace Profilers: Proposal," Light Source Note LSBL-800 (ALS, LBNL, Berkeley, October 17, 2006).

12. S. C. Irick. W. R. McKinney, "Advancements in one-dimensional profiling with a long trace profiler," SPIE Proc. 1720 (1992), 162-8.

13. S. C. Irick, W. R. McKinney, D. L. Lunt, P. Z. Takacs, "Using a straightness reference in obtaining more accurate surface profiles from a long trace profiler (for synchrotron optics)," Rev. Sci. Instrum., 63(1), 1436-8 (1992).

14. S. C. Irick, "Improved measurement accuracy in a long trace profiler: compensation for laser pointing instability," Nucl. Instrum. \& Meth. A 347(1-3), 226-30 (1994).

15. S. C. Irick, "Determine surface profile from sequential interference patterns from a long tracer profiler (for synchrotron optics)," Rev. Sci. Instrum. 63(1) (1992) 1432-5.

16. Shinan Qian, H. Li, P. Z. Takacs, "Penta-Prism Long Trace Profiler (PPLTP) for measurement of grazing incidence space optics," SPIE Proc. 2805 (1996), 108-14.

17. P. Z. Takacs, Shinan Qian, T. Kester, H. Li, "Large-mirror figure measurement by optical profilometry techniques," SPIE Proc. 3782 (1999), 266-74.

18. Shinan Qian, G. Sostero, P. Z. Takacs, "Precision calibration and systematic error reduction in the Long Trace Profiler," SPIE Proc. 3782 (1999), 627-36. 
19. Shinan Qian, Sostero G, P.Z. Takacs, "Precision calibration and systematic error reduction in the long trace profiler," Optical Engineering, 39(1) (2000), 304-10.

20. P. Z. Takacs, Shinan Qian, “Accuracy limitations in long-trace profilometry,” AIP Conference Proceedings, 708, 831-4 (2004).

21. Shinan Qian, W. Jark, P. Z. Takacs, "The penta-prism LTP: a long-trace-profiler with stationary optical head and moving penta prism," Rev. Sci. Instrum. 66(3) (1995), 2562-9.

22. P. Z. Takacs, C. J. Bresloff, "Significant improvements in long trace profiler measurement performance," SPIE Proc. 2856, 236-45 (1996).

23. P. Z. Takacs, E. L. Church, C. J. Bresloff, L. Assoufid, "Improvements in the accuracy and the repeatability of long trace profiler measurements," Applied Optics, 38(25), 5468-79 (1999).

24. Shinan Qian, P.Z. Takacs, "Equal optical path beam splitter for a pencil beam interferometer and shearing interferometer," Optical Engineering 42(4), 929-34 (2003).

25. V. V. Yashchuk, S. C. Irick, A. A. MacDowell, "Elimination of 'ghost'-effect-related systematic errors in metrology of X-ray optics with a long trace profiler," SPIE Proc. 5858, 261-268 (2005).

26. V.V. Yashchuk, "Positioning Errors of Pencil-beam Interferometers for Long Trace Profilers," SPIE Proc. 6317-10, (2006).

27. V. V. Yashchuk, S. C. Irick, A. A. MacDowell, W. R. McKinney, P. Z. Takacs, "Air convection noise of pencilbeam interferometer for long-trace profiler," SPIE Proc. 6317-13, 2006.

28. R. G. Geckeler, "ESAD shearing deflectometry: potentials for synchrotron beamline metrolog," SPIE Proc. 6317, 63171H (2006).

29. Melles Griot 57 ICS-Series Fiber-Coupled $670 \mathrm{~nm}, 3 \mathrm{~mW}$, Diode Lasers Module (http://lasers.mellesgriot.com/ Specsheet.asp?CatID=11521).

30. Applied Geomechanics 755-1172 Miniature Tilt Sensors (http://www.geomechanics.com/dspproduct.cfm?prid=1).

31. J. L. Kirschman, E. E. Domning, G. Y. Morrison, B. V. Smith, V. V. Yashchuk, "Precision Tiltmeter as a Reference for Slope Measuring Instruments," SPIE Proc. 6704-08 (2007) - this volume.

32. Applied Geomechanics Technical Note: Acceleration Effects on Tiltmeter Measurements (www.geomechanics.com/ dspapp.cfm?appid=85).

33. J. L. Kirschman, E. E. Domning, K. D. Franck, S. C. Irick, A. A. McDowell, W. R. McKinney, G. Y. Morrison, B. V. Smith, T. Warwick, V. V. Yashchuk, "Flat-field calibration of CCD detector for Long Trace Profiler," SPIE Proc. 6704-18 (2007) - this volume.

34. S. C. Irick, "Angle Calibration of the Long Trace Profiler Using a Diffraction Grating," LSBL Report LSBL-160 (1992).

35. F. Siewert, "Calibration: Autocalibration and Calibration," on $3^{\text {rd }}$ International Workshop on Metrology for X-ray Optics, Daegu, Korea, June 2006.

36. Daniel Boehlek "Erprobung einer Winkelkalibriereinheit für die Nanometer- Optikkomponete- Messmaschine (NOM)," Diplomarbeit, Berlin 2004.

37. F. Siewert, H. Lammert, T. Noll, T. Schelegel, T. Zeschke, T. Hansel, A. Nickel, A. Schindler, B. Grubert, C. Schlewitt, "Advanced metrology, an essential support for the surface finishing of high performance x-ray optics," SPIE Proc. 5921-01 (2005).

38. Physik Instrumente L.P., model M-227 (Physik Instrumente GmbH \& Co: www.physikinstrumente.com).

39. Heidenhain CERTO, model CP60K (HEIDENHAIN Co.: www.heidenhain.com). 